\title{
Ultrasonic Assessment of Females with Carpal Tunnel Syndrome Proved by Nerve Conduction Study
}

\author{
Ihsan M. Ajeena, ${ }^{1}$ Raed H. Al-Saad, ${ }^{2}$ Ahmed Al-Mudhafar, ${ }^{3}$ \\ Najah R. Hadi, ${ }^{3}$ and Sawsan H. Al-Aridhy ${ }^{2}$ \\ ${ }^{1}$ Neurophysiology Unit, Physiology Department, Faculty of Medicine, Kufa University, Najaf, Iraq \\ ${ }^{2}$ Radiology Unit, Surgery Department, Faculty of Medicine, Kufa University, Najaf, Iraq \\ ${ }^{3}$ Pharmacology Department, Faculty of Medicine, Kufa University, Najaf, Iraq \\ Correspondence should be addressed to Ihsan M. Ajeena; ihsanabud@yahoo.com
}

Received 25 March 2013; Revised 30 May 2013; Accepted 4 June 2013

Academic Editor: Preston E. Garraghty

Copyright (C) 2013 Ihsan M. Ajeena et al. This is an open access article distributed under the Creative Commons Attribution License, which permits unrestricted use, distribution, and reproduction in any medium, provided the original work is properly cited.

\begin{abstract}
Introduction. Carpal tunnel syndrome (CTS) is the most commonly diagnosed entrapment neuropathy of the upper extremity. The objective of this study was to diagnose CTS and to assess its severity using high resolution ultrasound (HRUS) depending on the results of nerve conduction study (NCS). Methods. A prospective cross-sectional study, in which HRUS was performed at 63 wrists of 35 female patients with different severity of CTS (as proved by NCS). Furthermore, 40 healthy volunteers ( 80 wrists) underwent the same tests as the patients and have been chosen to match the patients in gender, age, and body mass index (BMI). The cross section area (CSA) of the median nerve (MN) was obtained using HRUS at the carpal tunnel inlet by direct tracing method. Results. There was a significant difference in the CSA of the MN at the tunnel inlet in CTS patients when compared with the control group. In fact, the CSA of the control group showed a significant difference from each of patients subgroups. Furthermore, a significant difference in the CSA was seen in between these subgroups. In conclusion, the US examination of the MN seems to be a promising method in diagnosing and grading of carpal tunnel syndrome.
\end{abstract}

\section{Introduction}

Carpal tunnel syndrome (CTS) or compression neuropathy of the median nerve $(\mathrm{MN})$ at the wrist is the most common form of peripheral entrapment neuropathy $[1,2]$. It accounts for $90 \%$ of all entrapment neuropathies [3] and it is particularly prevalent in middle-aged women [4] and is recognized as one of the most important causes of the workplace morbidity [5]. The prevalence of CTS in the United Kingdom was $7-16 \%$ in 2010 , while in the United States was only $5 \%$ [6]. The diagnosis of CTS involves combination of a detailed clinical history, accurate examination, and appropriate electrodiagnostic studies (EDS) [7].

High resolution ultrasound (HRUS) has emerged as a feasible, simple, relatively low-cost, rapid, accurate, and noninvasive imaging method for evaluating the $\mathrm{MN}$ in the carpal tunnel $[1,2,4,5,8-21]$. Despite that, some authors consider that the role of ultrasound scanning (US) in diagnosis of CTS is yet to be proven [22] and other stated that US appears to be of little use in the diagnosis of CTS [9]. By contrast, Wong et al. [10] proposed an algorithm involving initial US examination of patients suspected of having CTS and secondary EDS performed only when US results were negative. Furthermore, some studies stated that US could be used to grade the severity of CTS [18]. In addition to the detection of increased cross sectional area (CSA) of $\mathrm{MN}$ in patients with CTS, US may be used to detect space-occupying lesions as ganglia, fibromata, neural tumors, and tenosynovitis that usually cause CTS symptoms [9]. A previous prospective study compared the diagnostic utility of US versus EDS and found equivalent sensitivities between the two techniques [14]. Magnetic Resonance Imaging (MRI) has been shown to have a role where rare causes for CTS are suspected and also in the detailed reconstruction of the anatomy to aid endoscopic 


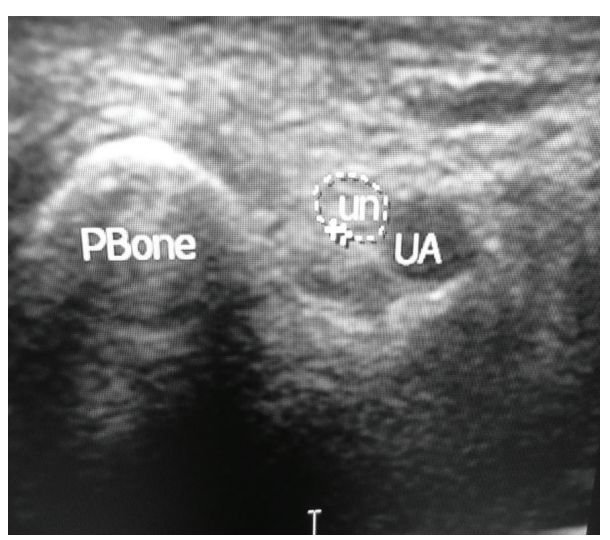

(a)

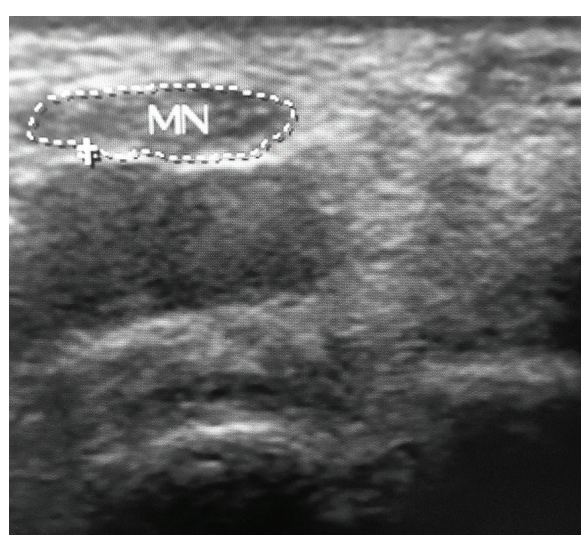

(b)

FIGURE 1: Wrist ultrasound at the carpal tunnel inlet for patient with mild CTS: (a) medial and (b) lateral aspects of the wrist (the tracing method for measuring the cross sectional area of the median nerve which was $10 \mathrm{~mm}^{2}$ ). UA: ulnar artery, UN: ulnar nerve in the Guyon canal, Pbone: pisiform bone, MN: median nerve.

procedures [22]. Hence, the objective of this study is to diagnose CTS and assess its severity using HRUS depending on the results of nerve conduction study (NCS).

\section{Materials and Methods}

A prospective cross-sectional study was employed at Al-Sadir Medical City in Al-Najaf health directorate. Forty female patients with a provisional diagnosis unilateral or bilateral CTS were selected randomly from the outpatient clinic of the Teaching Hospital and the duration of their clinical symptoms ranged from 2 months to 15 years. Of these, only 72 hands showed positive NCS findings documenting the presence of CTS. Furthermore, nine hands of 5 patients were excluded later on during US examination due to the presence of anatomical variations in the $\mathrm{MN}$ or space occupying lesion. The remaining 63 hands fulfill the criteria of this study and were analysed as patient group. Forty healthy volunteers with no clinical signs or symptoms of CTS and normal NCS findings were included as control group. These volunteers had been chosen to match the patients in gender, age, and body mass index (BMI). All the participants had no history of upper limb trauma, no systemic diseases such as rheumatoid arthritis, diabetes mellitus, and thyroid dysfunction. Pregnancy and provisional diagnosis of cervical radiculopathy were other exclusion criteria.

The study protocol was approved by the ethics committee of University of Kufa/Faculty of Medicine, and verbal consent was obtained from all patients and controls.

For NCS, the subjects were examined in the Middle Euphrates Neurosciences Center, AL-Sadir Medical City using Electromyoneurography instrument (Micromed System plus-EMG, Italian model 2001). Patients were diagnosed to have CTS through NCS by testing the sensory and motor fibers of both median and ulnar nerves bilaterally with recorded median nerve abnormal conduction parameters. The results subdivided the patient group into three subgroups; mild (Grade 2 = only sensory fibers involvement), moderate
(Grade $3 \& 4=$ in additional to grade 2, motor fibers involvement) and severe (Grade 5 \& $6=$ severe motor fibers involvement), according to the local severity scale of the neurophysiological reference values [23].

The US examination was achieved using HD11XE Philips 2009 and the US unit equipped with a broadband $3-12 \mathrm{MHz}$ linear transducer. The US evaluation was performed by the senior radiologist with special interest in musculoskeletal imaging who was blind to the degree of CTS severity reported by NCS at the time of the US study. Subjects were seated facing the examiner with their extended, supinated forearms, wrists were supported in a neutral position, and the fingers of that hand were semiextended. Ultrasonic gel was applied on the US probe to act as a coupling agent and then a real time transverse imaging of the MN from the distal forearm to the outlet of the carpal tunnel was performed. The CSA measurement of the MN was obtained using the standard protocol described by Duncan et al. [1] and Alemán et al. [24]. The technique of this protocol was as follows: (1) the transducer was positioned perpendicular to the $\mathrm{MN}$, with no pressure on the skin to avoid deformation of the nerve; (2) axial images were obtained at the level of the pisiform bone, and the image with the optimal definition of the borders of the MN was selected; and (3) MN CSA measurements were performed from the inner border of the perineural echogenic rim, corresponding to the perineurium around the hypoechoic MN. Measurement was performed using the direct method, a direct tracing with electronic calipers around the margin of the nerve (Figures 1, 2, and 3).

Descriptive statistics of the measured CSA of the MN at the carpal tunnel inlet were presented as mean \pm SD. Statistical analysis was carried out using Student's $t$-test and oneway ANOVA (Analysis of Variance) models to test differences between groups' means of continuous quantitative variables. The significance level ( $P$ value) $P<0.05$ was considered as statistically significant. Data manipulation and analysis were performed using the Statistical Package of Social Sciences (SPSS) version 19 software. 


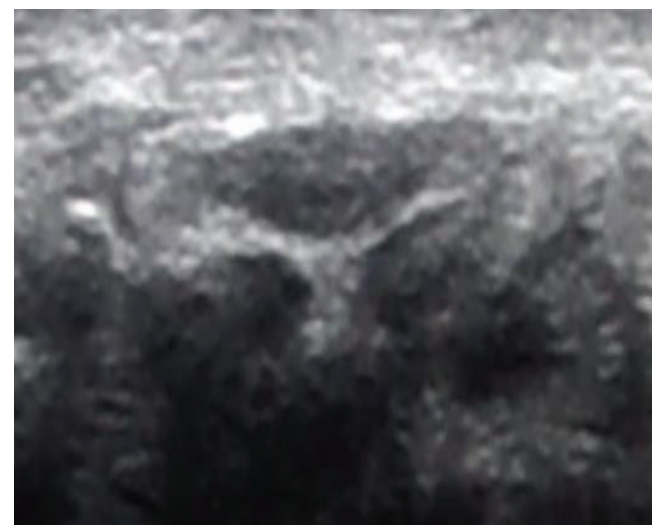

FIGURE 2: Wrist ultrasound at the carpal tunnel inlet for patient with moderate CTS, the cross sectional area of the median nerve was $14 \mathrm{~mm}^{2}$.

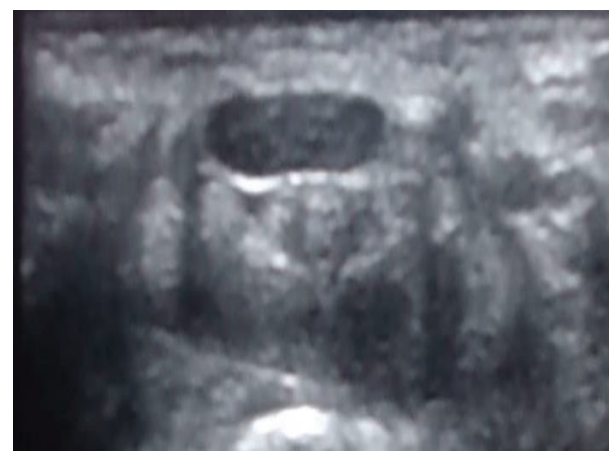

(a)

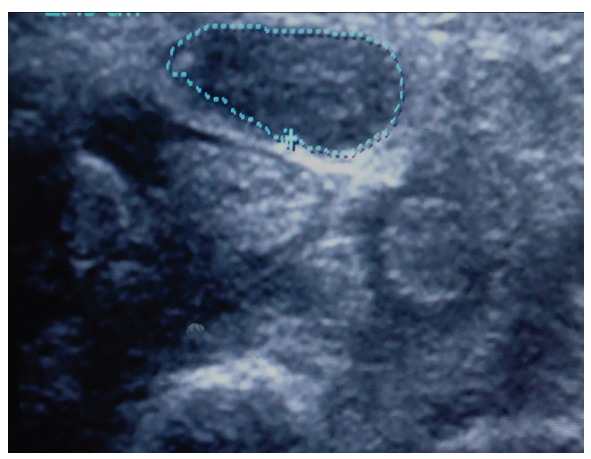

(b)

FIGURE 3: (a) Wrist ultrasound at the carpal tunnel inlet for patient with severe CTS medial, (b) the tracing method for measuring the cross sectional area of the median nerve which was $18 \mathrm{~mm}^{2}$.

\section{The Results}

The study population included 35 women with CTS as patient group and 40 volunteer women as control group. Both groups were matched in age and BMI (Table 1).

Only $28(80 \%)$ of the 35 patients had bilateral CTS, while the remaining 7 patients (20\%) had unilateral CTS. The dominant hand was affected in all of the unilateral cases. Of these 63 diseased wrists, 25 (40\%) showed mild, 27 (43\%) showed moderate, and $11(17 \%)$ showed severe CTS according to electrophysiologic results.
TABLE 1: The mean, standard deviation and range of age and BMI for the patient and control groups.

\begin{tabular}{lccccc}
\hline \multirow{2}{*}{ Sample } & \multirow{2}{*}{ Number } & \multicolumn{2}{c}{ Age/year } & \multicolumn{2}{c}{ BMI $\left(\mathrm{kg} / \mathrm{m}^{2}\right)$} \\
& & Mean \pm SD & Range & Mean \pm SD & Range \\
\hline $\begin{array}{l}\text { Patients } \\
\text { with CTS }\end{array}$ & 35 & $41.5 \pm 6.5$ & $31-50$ & $30.1 \pm 4.8$ & $23.5-38.1$ \\
Controls & 40 & $37 \pm 6.1$ & $30-53$ & $29.6 \pm 3.7$ & $23.4-36.6$ \\
$P$ value & - & $>0.05$ & - & $>0.05$ & - \\
\hline
\end{tabular}

CTS: carpal tunnel syndrome, BMI: body mass index, SD: standard deviation.

The CSA of the MN at the tunnel inlet (at the level of the pisiform bone) in the patients group was significantly greater than that of the control group (Table 2).

There was a significant difference of CSA of MNs of different patient subgroups (mild, moderate, and severe) when compared to the control group (Table 3). Furthermore, a significant difference of this CSA was also noted between these subgroups when compared with each other (Table 4).

\section{Discussion}

In this study, a logical occasion that CTS present in mean age of $(41.5 \pm 6.5)$ years agrees with other researchers as Phalen [25] who reported that the peak age range of patients with CTS was 40-60 years [26] and Akcar et al. [4] who studied a sample with ages ranging between 33 and 58 years.

On comparing the BMI of participating patients $(30.1 \pm$ $\left.4.8 \mathrm{~kg} / \mathrm{m}^{2}\right)$ with that of the control group $(29.6 \pm 3.7)$, the results showed no significant difference. It is known that a change in the BMI might affect the integrity of the nerve, a fact proved by many researchers as Werner et al. [27] who concluded that obese individuals (BMI > 29) are 2.5 times more likely to complain of CTS than slender individuals (BMI < 20). The correlation between CSA of the MN with BMI and hand physiognomies (small or strong wrists) may exist [9] and by 2012, Jessie et al. found that BMI had the greatest impact on ulnar nerve size [28]. That is why the control group of this study was selected to match the patients in regard to their BMI.

The participants were all females to avoid the effect of gender on results of the CSA as Andrea et al. recoded that CSA of the MN proximal to carpal tunnel was greater in men than in women by $2.2 \mathrm{~mm}^{2}$ [9].

The results revealed a significant increase of the CSA of MNs at the tunnel inlet in the patient group (13.11 \pm $\left.3 \mathrm{~mm}^{2}\right)$ when compared with that of the control group $(6.87 \pm$ $1.04 \mathrm{~mm}^{2}$ ), a finding that is consistent with that of Akcar et al. [4]. At the same time, a significant difference in between three patients subgroups and with the control group was also recorded $(P<0.001)$.

It is not known exactly whether neuropathy of the MN develops as a result of intermittent mechanic compression or as a result of vascular compromise due to a rise in intracranial pressure [29], and perhaps both are responsible for the progression of CTS. Vascular compromise seems to occur in 
TABLE 2: The descriptive statistics for CSA of MNs of patients and controls at the carpal tunnel inlet.

\begin{tabular}{lccccc}
\hline \multirow{2}{*}{ Sample } & \multirow{2}{*}{ No. of wrists } & \multirow{2}{*}{ CSA (mean \pm SD) } & \multicolumn{2}{c}{$95 \%$ Confidence interval for mean } & \multirow{2}{*}{ Minimum } \\
& & & Lower bound & Upper bound & \\
\hline Patients with CTS & 63 & $13.11 \pm 3.074$ & 12.33 & 13.89 & 8.10 \\
Control & 80 & $6.87 \pm 1.041$ & 6.64 & 7.10 & 20.00 \\
$P$ value & & $<0.001$ & & & 8.50 \\
\hline
\end{tabular}

CSA: cross sectional area of median nerve, CTS: carpal tunnel syndrome, SD: standard deviation.

TABLE 3: The CSA of MNs for the different patient subgroups versus that of the control group (depending on the results of the nerve conducting study).

\begin{tabular}{|c|c|c|c|c|c|}
\hline \multirow{2}{*}{ CSA at the tunnel inlet $\left(\mathrm{mm}^{2}\right)$} & \multicolumn{3}{|c|}{ Subgroups of CTS patients according to NCS } & \multirow{2}{*}{ Control $(n=80)$} & \multirow{2}{*}{$P$ value } \\
\hline & Mild $(n=25)$ & Moderate $(n=27)$ & Severe $(n=11)$ & & \\
\hline & $10.26 \pm 0.83$ & $13.81 \pm 1.62$ & $17.86 \pm 1.89$ & $6.87 \pm 1.04$ & $<0.001$ \\
\hline
\end{tabular}

CSA: cross sectional area of the median nerve, CTS: carpal tunnel syndrome, NCS: nerve conduction study.

TABLE 4: Multiple comparisons of CSA of the MNs of different patient subgroups with each other and with that of the control group.

\begin{tabular}{lccc}
\hline Group type & Remaining groups & Mean difference & Significance \\
\hline \multirow{3}{*}{ Mild CTS } & Moderate & -3.55081 & $<0.001$ \\
& Severe & -7.59964 & $<0.001$ \\
& Control & 3.39275 & $<0.001$ \\
\hline \multirow{3}{*}{ Moderate CTS } & Mild & 3.55081 & $<0.001$ \\
& Severe & -4.04882 & $<0.001$ \\
& Control & 6.94356 & $<0.001$ \\
\hline \multirow{2}{*}{ Severe CTS } & Mild & 7.59964 & $<0.001$ \\
& Moderate & 4.04882 & $<0.001$ \\
& Control & 10.99239 & $<0.001$ \\
\hline
\end{tabular}

CSA: cross sectional area, MNs: median nerves, CTS: carpal tunnel syndrome.

three stages: venous congestion; nerve edema, and impairment of the venous-arterial blood supplies [30]. A rise in pressure in carpal tunnel above normal, 20 to $30 \mathrm{mmHg}$, causes a chronic compressive ischemic injury to the nerve segment, resulting first in demyelination and eventually in axonal death. This will cause a progressive conduction block in the nerve with subsequent sensory and motor dysfunction [31]. When pressure builds on the median nerve, the blood supply to the outer covering of the nerve slows down and may even be cut off, causing ischemia. At first, only the outside covering of the nerve is affected but if the pressure keeps building up, the inside of the nerve will start to become thickened. New cells (fibroblasts) form within the nerve and create scar tissue [32].

The results of this study were in agreement with the results obtained by Karadag et al., who stated that the US was useful in grading the severity of CTS. They concluded that US measurement of CSA could give information about severity of MN involvement and they set US cut-off points that discriminate between different grades of CTS severity as follows: $10.0-13.0 \mathrm{~mm}^{2}$ for mild, $13.0-15.0 \mathrm{~mm}^{2}$ for moderate, and $>15.0 \mathrm{~mm}^{2}$ for severe symptoms [18]. Also, El Miedany et al. [11] and Lee et al. [33] found that one can be confident of determining the level of severity of CTS based on US measurement of CSA of the MNs. In their work, they reported that US measurements of greater than $15 \mathrm{~mm}^{2}$ correlate with NCS findings of moderate to severe disease and noted that these figures differ significantly from those patients with mild to moderate disease. Furthermore, Moran et al. reported that the CSA of the MN at the tunnel inlet were $10.8 \pm 1.9 \mathrm{~mm}^{2}, 11.4 \pm$ $1.8 \mathrm{~mm}^{2}$, and $12 \pm 1.5 \mathrm{~mm}^{2}$ in patients with mild, moderate, and severe CTS, respectively. They reported that their clinical groups differed significantly from their control group (5.8 \pm $0.9 \mathrm{~mm}^{2}$ ), but they found no differences between the patient groups [5]. This further underscores one of our principal findings. In even starker contrast to the present findings, Mohammadi et al. asserted that US cannot be used to grade the severity of CTS [8].

In the current study, the CAS of the MN was measured directly with electronic calipers around the margin of the nerve. This strategy has been employed in a number of studies that have reported that the direct method has greater diagnostic reliability than the indirect method (ellipsoid formula) $[1,2,10,11,16]$.

Our results are consistent with previous reports that demonstrated the utility of US measurement of MN CSA at the tunnel inlet as a good alternative to NCS for the initial diagnosis of CTS [7, 10]. Furthermore, several other studies had concluded that CSA is the most predictive measurement for the diagnosis of CTS $[2,16,17]$. An interesting fact is that many studies showed the lack of interreader reliability of the CSA measurements obtained at the tunnel outlet [5] because MN may be difficult to be seen at outlet in persons with thick palmar skin and it has a wide variation as it usually splits into digital branches here [10]. That is why the current study used measurements of the CSA of the MN at the tunnel inlet despite the findings of Mohammadi et al., in 2009 about the usefulness of measuring CSA of the MN at the tunnel outlet [8].

In conclusion, the $\mathrm{MN}$ is easily visualized and measuring its CSA at the level of pisiform bone using HRUS is a sensitive, specific, and useful noninvasive method for the diagnosis of CTS. Furthermore, this diagnostic method is a reliable test 
in assessing the severity of CTS and might reveal some of its possible causes as space occupying lesion or anatomical variation of the MN. Finally, US examination of MNs seems to be a promising method for diagnosing and grading CTS.

\section{Conflict of Interests}

The authors declare that there is no conflict of interests related to this paper.

\section{Acknowledgments}

The authors would like to express their gratitude to the patients and volunteers who participated in this study and to the staff of neurophysiology and radiology units at the hospital and to all colleagues who supported and facilitated this work. Also, they owe acknowledgments to the anonymous reviewers of initial submitted paper for their helpful comments. These acknowledgments extend to the editors for their patience and encouragements that helped them come out with this paper as such.

\section{References}

[1] I. Duncan, P. Sullivan, and F. Lomas, "Sonography in the diagnosis of carpal tunnel syndrome," American Journal of Roentgenology, vol. 173, no. 3, pp. 681-684, 1999.

[2] L. Sarría, T. Cabada, R. Cozcolluela, T. Martínez-Berganza, and S. García, "Carpal tunnel syndrome: usefulness of sonography," European Radiology, vol. 10, no. 12, pp. 1920-1925, 2000.

[3] S. Aroori and R. A. J. Spence, "Carpal tunnel syndrome," Ulster Medical Journal, vol. 77, no. 1, pp. 6-17, 2008.

[4] N. Akcar, S. Özkan, Ö. Mehmetoglu, C. Calisir, and B. Adapinar, "Value of power Doppler and gray-scale US in the diagnosis of carpal tunnel syndrome: contribution of cross-sectional area just before the tunnel inlet as compared with the cross-sectional area at the tunnel," Korean Journal of Radiology, vol. 11, no. 6, pp. 632-639, 2010.

[5] L. Moran, M. Perez, A. Esteban, J. Bellon, B. Arranz, and M. Del Cerro, "Sonographic measurement of cross-sectional area of the median nerve in the diagnosis of carpal tunnel syndrome: correlation with nerve conduction studies," Journal of Clinical Ultrasound, vol. 37, no. 3, pp. 125-131, 2009.

[6] S. E. Luckhaupt, J. M. Dahlhamer, B. W. Ward, M. H. Sweeney, J. P. Sestito, and G. M. Calvert, "Prevalence and work-relatedness of carpal tunnel syndrome in the working population, United States, 2010 national health interview survey," American Journal of Industrial Medicine, vol. 55, no. 12, pp. 1069-1077, 2012.

[7] D. Rempel, B. Evanoff, P. C. Amadio et al., "Consensus criteria for the classification of carpal tunnel syndrome in epidemiologic studies," American Journal of Public Health, vol. 88, no. 10, pp. 1447-1451, 1998.

[8] A. Mohammadi, A. R. Afshar, S. Masudi, and A. Etemadi, "Comparison of high resolution ultrasonography and nerve conduction study in the diagnosis of carpal tunnel syndrome: diagnostic value of median nerve cross-sectional area," Iranian Journal of Radiology, vol. 6, no. 3, pp. 147-152, 2009.

[9] S. Andrea, J. Ethan, F. Ralph et al., "Bifid median nerve in carpal tunnel syndrome: assessment with US cross-sectional area measurement," Radiology, vol. 259, no. 3, pp. 123-129, 2011.
[10] S. M. Wong, J. F. Griffith, A. C. F. Hui, S. K. Lo, M. Fu, and K. S. Wong, "Carpal tunnel syndrome: diagnostic usefulness of sonography," Radiology, vol. 232, no. 1, pp. 93-99, 2004.

[11] Y. M. El Miedany, S. A. Aty, and S. Ashour, "Ultrasonography versus nerve conduction study in patients with carpal tunnel syndrome: substantive or complementary tests?" Rheumatology, vol. 43, no. 7, pp. 887-895, 2004.

[12] W. Buchberger, G. Schon, K. Strasser, and W. Jungwirth, "Highresolution ultrasonography of the carpal tunnel," Journal of Ultrasound in Medicine, vol. 10, no. 10, pp. 531-537, 1991.

[13] W. Buchberger, W. Judmaier, G. Birbamer, M. Lener, and C. Schmidauer, "Carpal tunnel syndrome: diagnosis with highresolution sonography," American Journal of Roentgenology, vol. 159, no. 4, pp. 793-798, 1992.

[14] M. Mondelli, G. Filippou, A. Gallo, and B. Frediani, "Diagnostic utility of ultrasonography versus nerve conduction studies in mild carpal tunnel syndrome," Arthritis Care and Research, vol. 59, no. 3, pp. 357-366, 2008.

[15] M. G. Hochman and J. L. Zilberfarb, "Nerves in a pinch: imaging of nerve compression syndromes," Radiologic Clinics of North America, vol. 42, no. 1, pp. 221-245, 2004.

[16] S. M. Wong, J. F. Griffith, A. C. F. Hui, A. Tang, and K. S. Wong, "Discriminatory sonographic criteria for the diagnosis of carpal tunnel syndrome," Arthritis and Rheumatism, vol. 46, no. 7, pp. 1914-1921, 2002.

[17] L.-Y. Wang, C.-P. Leong, Y.-C. Huang, J.-W. Hung, S.-M. Cheung, and Y.-P. Pong, "Best diagnostic criterion in highresolution ultrasonography for carpal tunnel syndrome," Chang Gung Medical Journal, vol. 31, no. 5, pp. 469-476, 2008.

[18] Y. S. Karadag, O. Karadag, and E. Cicekli, "Severity of carpal tunnel syndrome assessed with high frequency ultrasonography," Rheumatology International, vol. 30, no. 6, pp. 761-765, 2010.

[19] I. K. Keleş, A. T. Kendi, G. Aydin, S. G. Zöğ, and S. Orkun, "Diagnostic precision of ultrasonography in patients with carpal tunnel syndrome," American Journal of Physical Medicine of Rehabilitation, vol. 84, pp. 443-450, 2005.

[20] A. S. Klauser, E. J. Halpern, T. De Zordo et al., "Carpal tunnel syndrome assessment with US: value of additional crosssectional area measurements of the median nerve in patients versus healthy volunteers," Radiology, vol. 250, no. 1, pp. 171-177, 2009.

[21] M. Keberle, M. Jenett, W. Kenn et al., "Technical advances in ultrasound and MR imaging of carpal tunnel syndrome," European Radiology, vol. 10, no. 7, pp. 1043-1050, 2000.

[22] S. P. Mathew, P. Jonathan, S. K. Wasim, and J. Nicholas, "Is there light at the end of the tunnel? Controversies in the diagnosis and management of carpal tunnel syndrome," American Association For Hand Surgery, vol. 5, no. 4, pp. 354-360, 2010.

[23] J. D. Bland, "A neurophysiological grading scale for carpal tunnel syndrome," Muscle \& Nerve, vol. 23, no. 8, pp. 1280-1283, 2000.

[24] L. Alemán, J. D. Berná, M. Reus, F. Martínez, G. DoménechRatto, and M. Campos, "Reproducibility of sonographic measurements of the median nerve," Journal of Ultrasound in Medicine, vol. 27, no. 2, pp. 193-197, 2008.

[25] G. S. Phalen, “The carpal-tunnel syndrome. Seventeen years' experience in diagnosis and treatment of six hundred fifty-four hands," Journal of Bone and Joint Surgery A, vol. 48, no. 2, pp. 211-228, 1966. 
[26] J. W. Hilburn, "General principles and use of electrodiagnostic studies in carpal and cubital tunnel syndromes: with special attention to pitfalls and interpretation," Hand Clinics, vol. 12, no. 2, pp. 205-221, 1996.

[27] R. A. Werner, J. W. Albers, A. Franzblau, and T. J. Armstrong, "The relationship between body mass index and the diagnosis of carpal tunnel syndrome," Muscle and Nerve, vol. 17, no. 6, pp. 632-636, 1994.

[28] T. Jessie, P. Maureen, and A. Kerry, "Impact of ancestry and body size on sonographic ulnar nerve dimensions," Radiography, vol. 18, no. 2, pp. 100-104, 2012.

[29] T. F. Elwakil, A. Elazzazi, and H. Shokeir, "Treatment of carpal tunnel syndrome by low-level laser versus open carpal tunnel release," Lasers in Medical Science, vol. 22, no. 4, pp. 265-270, 2007.

[30] A. Mallouhi, P. Pültzl, T. Trieb, H. Piza, and G. Bodner, "Predictors of carpal tunnel syndrome: accuracy of gray-scale and color doppler sonography," American Journal of Roentgenology, vol. 186, no. 5, pp. 1240-1245, 2006.

[31] M. Courtney and J. Townsend, Sabiston Textbook of Surgery, Saunders Elsevier, Toronto, Canada, 18th edition, 2007.

[32] J. Pomerance and I. Fine, "Outcomes of carpal tunnel surgery with and without supervised postoperative therapy," Journal of Hand Surgery, vol. 32, no. 8, pp. 1159-1163, 2007.

[33] D. Lee, M. T. Van Holsbeeck, P. K. Janevski, D. L. Ganos, D. M. Ditmars, and V. B. Darian, "Diagnosis of carpal tunnel syndrome: ultrasound versus electromyography," Radiologic Clinics of North America, vol. 37, no. 4, pp. 859-872, 1999. 

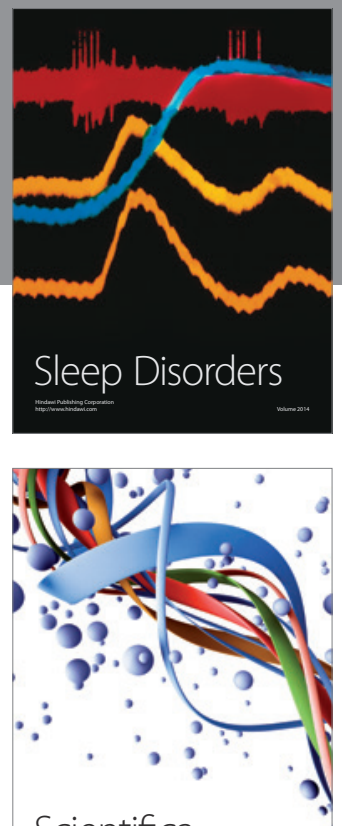

Scientifica
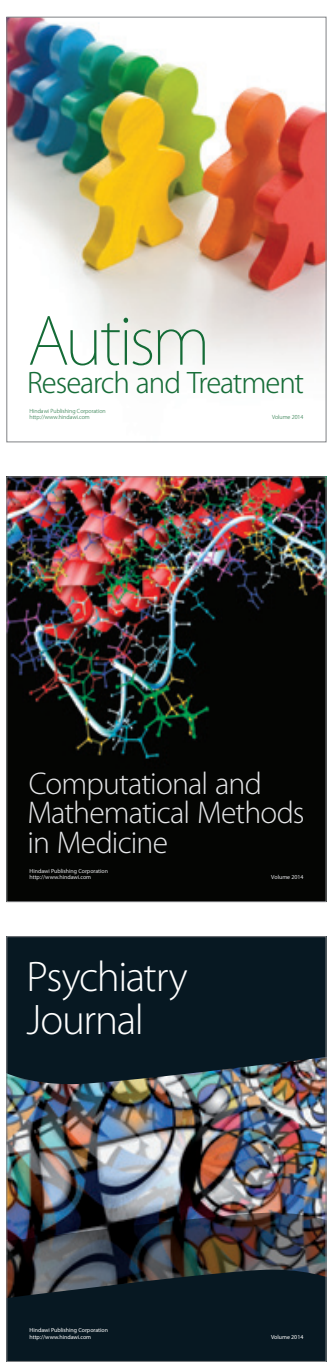
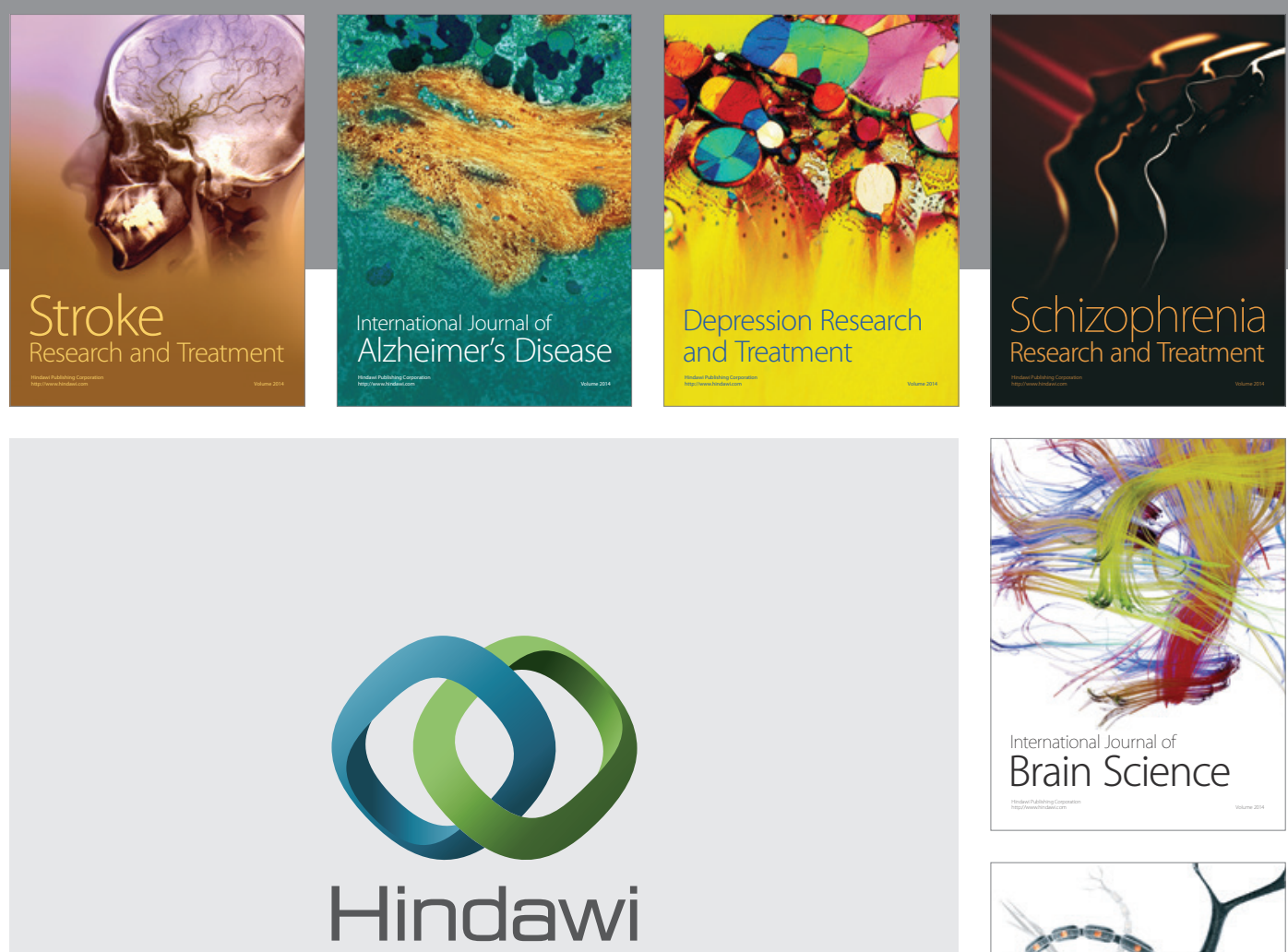

Submit your manuscripts at

http://www.hindawi.com
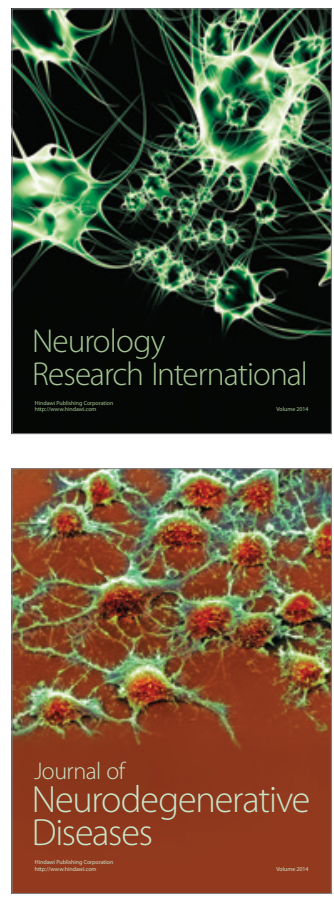

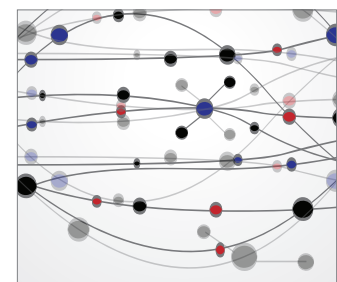

The Scientific World Journal
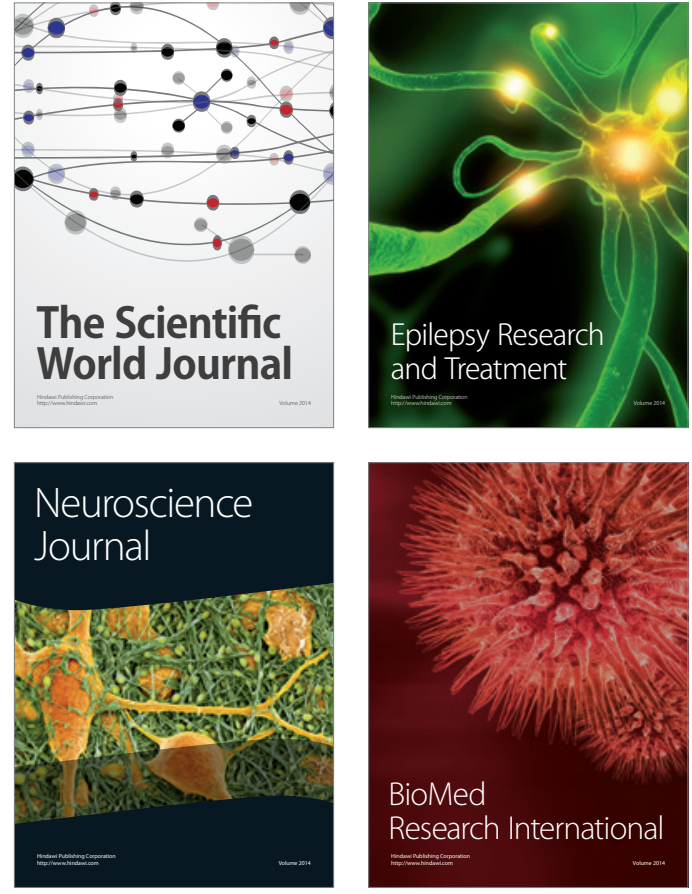

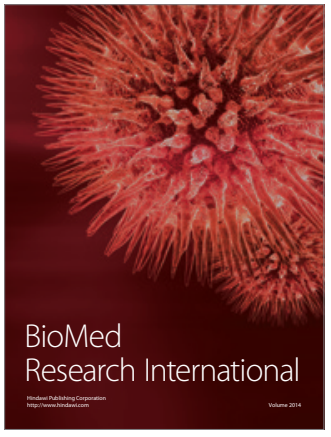

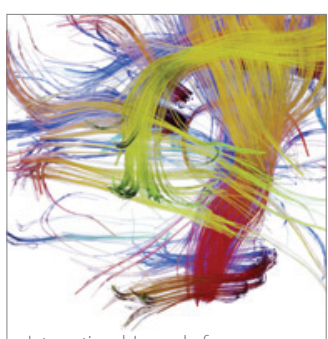

Brain Science

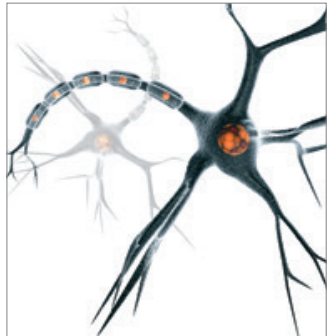

Neural Plasticity
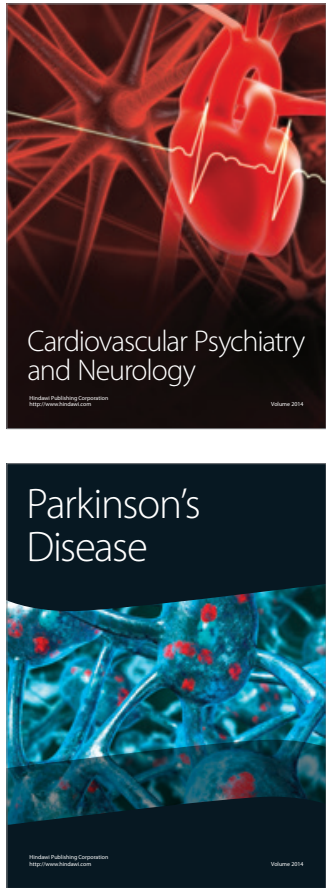